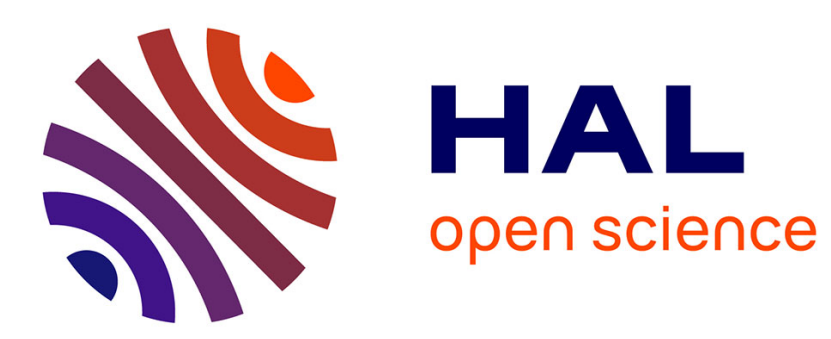

\title{
A free boundary problem for the Laplacian with constant Bernoulli-type boundary condition
}

\author{
Erik Lindgren, Yannick Privat
}

\section{To cite this version:}

Erik Lindgren, Yannick Privat. A free boundary problem for the Laplacian with constant Bernoullitype boundary condition. Nonlinear Analysis: Theory, Methods and Applications, 2007, 67 (8), pp.2497-2505. 10.1016/j.na.2006.08.045 . hal-00128760

\section{HAL Id: hal-00128760 \\ https://hal.science/hal-00128760}

Submitted on 2 Feb 2007

HAL is a multi-disciplinary open access archive for the deposit and dissemination of scientific research documents, whether they are published or not. The documents may come from teaching and research institutions in France or abroad, or from public or private research centers.
L'archive ouverte pluridisciplinaire HAL, est destinée au dépôt et à la diffusion de documents scientifiques de niveau recherche, publiés ou non, émanant des établissements d'enseignement et de recherche français ou étrangers, des laboratoires publics ou privés. 


\title{
A FREE BOUNDARY PROBLEM FOR THE LAPLACIAN WITH CONSTANT BERNOULLI-TYPE BOUNDARY CONDITION
}

\author{
ERIK LINDGREN AND YANNICK PRIVAT
}

\begin{abstract}
We study a free boundary problem for the Laplace operator, where we impose a Bernoulli-type boundary condition. We show that there exists a solution to this problem. We use A. Beurling's technique, by defining two classes of sub- and supersolutions and a Perron argument. We try to generalize here a previous work of A. Henrot and H. Shahgholian. We extend these results in different directions.
\end{abstract}

\section{INTRODUCTION}

1.1. The problem. The aim of this paper is to prove the existence and uniqueness of a Bernoulli-type free boundary problem in $\mathbb{R}^{n}$. Consider a smooth, bounded and convex domain $K$ such that $K \cap\left\{x_{1}=0\right\}$. We seek a bounded domain $\Omega \subset \mathbb{R}_{+}^{n}=\left\{\mathbb{R}^{n}: x_{1}>0\right\}$ with $K \subset \partial \Omega$, together with a function $u: \Omega \rightarrow \mathbb{R}$ such that

$$
\left\{\begin{array}{l}
\Delta u=0 \text { in } \Omega \\
u=1 \text { on } K \\
u=0 \text { on } \partial \Omega \backslash K \\
|\nabla u|=1 \text { on }(\partial \Omega \backslash K) \cap \mathbb{R}_{+}^{n} .
\end{array}\right.
$$

This problem arises from various areas, for instance shape-optimization, fluid dy-

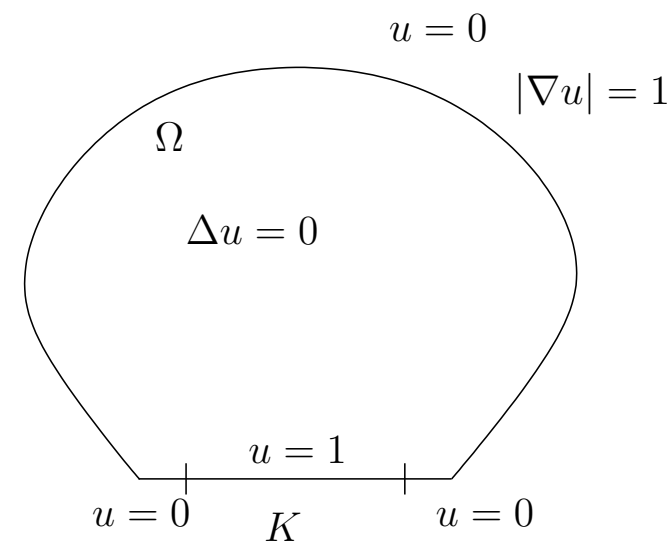

Figure 1. The geometric situation in $\mathbb{R}^{2}$.

namics, electrochemistry and electromagnetics. See for example [1], [3] and [5]. We also see a possibility to extend the results in $[2]$ and $[8]$ to be valid in our case.

Date: February 2, 2007 
1.2. The main theorem. The main theorem of the paper is:

Theorem 1. There is a unique solution to the free boundary problem $(\mathcal{P})$ with $\partial \Omega$ being $C^{2+\alpha}$ for any $0<\alpha<1$. In particular, the free boundary ( $\left.\partial \Omega \backslash K\right)$ meets the fixed boundary $(K)$ tangentially. Moreover, the solution has convex level sets.

1.3. Outline of the proof. The method used is as follows. Let $\mathcal{C}$ be the class of smooth, bounded and convex domains in $\mathbb{R}^{n}$ such that $K$ belongs to the boundary of the domain. Let $\Omega \in \mathcal{C}$, we denote furthermore by $u_{\Omega}$ the function fulfilling

$$
\left\{\begin{array}{l}
\Delta u_{\Omega}=0, \\
u_{\Omega}=0 \text { in } \partial \Omega \backslash K, \\
u_{\Omega}=1 \text { in } K .
\end{array}\right.
$$

Let us introduce the following classes of domains

$$
\begin{aligned}
\mathcal{A} & =\left\{\Omega \in \mathcal{C}: \liminf _{y \rightarrow x}\left|\nabla u_{\Omega}(y)\right| \geq 1, \forall x \in \partial \Omega \cap \mathbb{R}_{+}^{n}\right\}, \\
\mathcal{A}_{0} & =\left\{\Omega \in \mathcal{C}: \limsup _{y \rightarrow x}\left|\nabla u_{\Omega}(y)\right|>1, \forall x \in \partial \Omega \backslash K\right\}, \\
\mathcal{B} & =\left\{\Omega \in \mathcal{C}: \limsup _{y \rightarrow x}\left|\nabla u_{\Omega}(y)\right| \leq 1, \forall x \in \partial \Omega \backslash K\right\} .
\end{aligned}
$$

Translated into terms of $\mathcal{A}$ and $\mathcal{B}$, the aim of this project is to prove that $\mathcal{A} \cap \mathcal{B} \neq \emptyset$. To do this, we use Beurling's technique. In particular, we show that a subclass of $\mathcal{B}$ has, in some sense, a minimal element (if it is non-empty). This part of the proof relies mainly on Lemma 1 , the bound on $|\nabla u|$ (Lemma 2). These results are proved using the same arguments as in [6], [7] and [8].

In what follows we prove that the minimal element of $\mathcal{B}$ belongs to $\mathcal{A}$ as well. Mainly, we use Lemma 4 and some barrier arguments together with Lemma 1.

The proof is more or less a synthesis of [6] and [8]. The big difference in this problem is that the free boundary and the fixed boundary do meet. 


\section{Preliminaries}

Before we start treating the classes we need a bit of preparations. The results in this subsection are more or less already known, but not proved in detail for this particular case.

The first thing we prove is that the level sets of a Dirichlet solution are convex.

Theorem 2. Let $\Omega \in \mathcal{C}$. Then the level sets of $u_{\Omega}$, i.e. the sets $\mathcal{L}_{\varepsilon}=\{x \in \Omega$ : $\left.u_{\Omega}>\varepsilon\right\}$, are convex.

Proof. Let

$$
\begin{gathered}
K_{n}=\{x \in \Omega: \operatorname{dist}(x, K)<n / 2\} \\
\Omega_{n}=\{x \in \Omega: \operatorname{dist}(x, \Omega)<n\} .
\end{gathered}
$$

Then $D_{n}=\Omega_{n} \backslash K_{n}$ is a convex ring. Therefore, $u_{n}=u_{D_{n}}$ has convex level sets (cf [9]). By standard arguments, $u_{n}$ converges to a harmonic function in every compact subset of $\Omega$. Furthermore we will have $C^{0, \alpha}$-convergence in $\mathbb{R}^{n}$. Clearly, $u=1$ on $I$ and $u=0$ on $\mathbb{R}^{n} \backslash(\Omega \cup K)$. Hence, $u_{n} \rightarrow u_{\Omega}$. Moreover, $\mathcal{L}_{\varepsilon}=\cap \mathcal{L}_{\varepsilon}^{n}$, where $\mathcal{L}_{\varepsilon}^{n}=\left\{x \in \Omega: u_{n}>\varepsilon\right\}$, which implies that the sets $\mathcal{L}_{\varepsilon}$ are convex.

Now we prove a fundamental (but not trivial) lemma (based on the same idea than in [8]), which has some very important consequences when comparing the gradient on the boundary.

Lemma 1. We denote by $x_{1}$ the first coordinate in $\mathbb{R}^{n}$. Let $u=u_{\Omega}$ with $\Omega \in \mathcal{C}$, such that $u$ is Lipschitz on the boundary. Suppose that the gradient of $u$ exists on the boundary and that it is for every $r>0$ uniformly bounded by a constant $M(r)$ in $\{x: \operatorname{dist}(K, x)<r\}$. Then we will have, after suitable rotations and translations,

$$
u(x)=u\left(x^{0}\right)+\alpha\left(x_{1}-x_{1}^{0}\right)_{+}+o\left(r_{n}\right) \text { for } x_{0} \in \partial \Omega \backslash\{x: \operatorname{dist}(K, x)<r\},
$$

for some sequences $r_{n}$. In particular, we will have

$$
\limsup _{y \rightarrow x_{0}}|\nabla u(y)|=\limsup _{y \rightarrow x_{0}}\langle\nabla u(y), v\rangle \geq 0,
$$

where $v$ is a normal vector orthogonal the tangent plane at $x_{0}$ (or to one of them, if there are several).

Remark 1. We remark an immediate consequence of this theorem; let $u$ and $v$ be non-negative harmonic functions inside a domain $\Omega$ such that $u=v=0$ in some neighborhood of $x \in \partial \Omega$ and $u \geq v$ in $\Omega$. Then

$$
\limsup _{y \rightarrow x}|\nabla u(y)| \geq \limsup _{y \rightarrow x}|\nabla v(y)| .
$$

Indeed, apply Lemma 1 to the functions $u$ and $v$ and use that they both attain a minimum at $x$. The result follows immediately.

Now we just observe that $|\nabla u|^{2}$ is a subharmonic function if $u$ is harmonic. To finish up the preparations we show that the gradient is almost uniformly bounded on the boundary. The proof is more or less taken from [6].

Lemma 2. Let $\Omega \in \mathcal{C}$. Then the gradient of $u_{\Omega}$ is uniformly bounded outside and far enough from $K$, i.e. for each $r_{0}>0$ there is a constant $M\left(r_{0}\right)$ such that:

$$
|\nabla u| \leq M\left(r_{0}\right)
$$

for all $x \in \Omega \backslash N\left(r_{0}\right)$, where $N\left(r_{0}\right)=\left\{x: \operatorname{dist}(K, x)<r_{0}\right\}$.

Remark 2. In [2], the authors prove this lemma in a more simple case, in the sense that the convex domain $K$ doesn't belong to the boundary of $\Omega$. Lemma 3 prove that the gradient of $u_{\Omega}$ is bounded even if $K \cap \bar{\Omega} \neq \emptyset$. 
Proof. We observe that, by barrier arguments and the use of Lemma 1 we have that away from $K, \nabla u=0$ where $\partial \Omega$ is not $C^{1}$. So we can suppose that $\partial \Omega$ is $C^{1}$ away from $K$.

Since $|\nabla u|^{2}$ is subharmonic inside $\Omega$ it suffices thus to show that the gradient is bounded on $\partial\left(\Omega \backslash N\left(r_{0}\right)\right)$.

Let $r_{0}>0$ and let $\widehat{K}=K \backslash N\left(r_{0}\right)$.

(i) First Case: $x \in \widehat{K}$.

Take $B_{r_{0} / 3}$ and $B_{r_{0} / 2}$ be centered at $\left(x-r_{0} / 3 x_{2}\right)$. Consider now $\hat{u}$, the capacitary potential on $B_{r_{0} / 2} \backslash B_{r_{0} / 3}$, i.e. the harmonic function being zero on $\partial B_{r_{0} / 2}$ and one on $\partial B_{r_{0} / 3}$. Then we have by the comparison principle that $u \geq \widehat{u}$ inside $\left(B_{r_{0} / 2} \backslash B_{r_{0} / 3}\right) \cap \Omega$, which by Lemma 1 implies

$$
|\nabla u(x)| \leq|\nabla \widehat{u}(x)| \leq M\left(r_{0}\right) .
$$

We observe that we could repeat this for every point in $\widehat{K}$ without changing the radius of the balls. Hence, the inequality is valid for all $x \in \widehat{K}$.

(ii) Second Case: $\quad x \in \partial N\left(r_{0}\right) \cap \Omega$.

We use more or less the same arguments as above; let the balls $B_{r_{0} / 3}$ and $B_{r_{0} / 2}$ be centered at the same point such that $B_{r_{0} / 3} \subset\{u \leq u(x)\}$ and $\bar{B}_{r_{0} / 3} \cap\{u=u(x)\}=x$. This is possible since the level sets are by Theorem 2 convex. Let $\hat{u}$ denote the harmonic function inside $B_{r_{0} / 2} \backslash B_{r_{0} / 3}$ such that $\hat{u}=u(x)$ on $\partial B_{r_{0} / 3}$ and $\hat{u}=0$ on $\partial B_{r_{0} / 2}$. The comparison principle and Lemma 1 together imply

$$
|\nabla u(x)| \leq|\nabla \hat{u}(x)| \leq M\left(r_{0}\right) .
$$

Clearly, this inequality is valid for all $x \in \partial N\left(r_{0}\right) \cap \Omega$ since the function used will be the same.

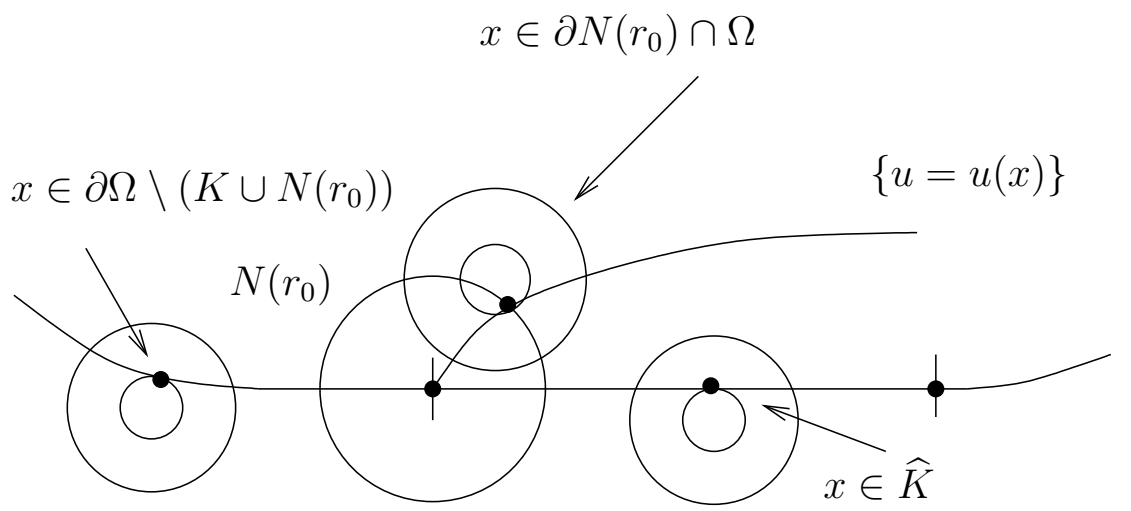

Figure 2. The picture when $n=2$.

(iii) Third Case: $x \in \partial \Omega \backslash\left(K \cup N\left(r_{0}\right)\right)$.

Let $B_{r_{0} / 3}$ and $B_{r_{0} / 2}$ be centered at the same point such that $\bar{B}_{r_{0} / 3} \cap \Omega=x$. We pick again a capacitary potential $\hat{u}$ on $B_{r_{0} / 2} \backslash B_{r_{0} / 3}$, such that $\hat{u}=0$ on $\partial B_{r_{0} / 3}$ and $\hat{u}=1$ on $\partial B_{r_{0} / 2}$. Then, as before, we obtain

$$
|\nabla u(x)| \leq|\nabla \hat{u}(x)| \leq M\left(r_{0}\right)
$$

uniformly.

The result follows. 
In the sequel we will use the following:

Proposition 1. Let $\Omega \in \mathcal{C}$ and $x \in \partial \Omega$. Then

$$
\liminf _{y \rightarrow x}\left|\nabla u_{\Omega}(x)\right|>0 \text {. }
$$

Proof. Using the exact same arguments as in the proof of Lemma 2 in [9] we obtain that for every $x \in \partial \Omega$ there is a $v$ such that

$$
\liminf _{y \rightarrow x}\left\langle\nabla u_{\Omega}(x), v\right\rangle>0,
$$

which immediately implies the desired result.

\section{Beurling's technique}

As mentioned earlier we revisit Beurling's technique to prove our main result. The arguments that we will use are more or less the same as the proofs of Henrot and Shahgholian in [6] and [8]. First, we give the steps of this technique and then, corresponding theorems.

- The class $\mathcal{B}$ is closed under intersection.

- Consequence: we consider a decreasing sequence of convex domains in $\mathcal{B}$. Then, the interior of the closure belongs to $\mathcal{B}$.

- We use this argument in order to construct a candidate for being the solution of our problem.

- Then, we check that this candidate is the solution of the free boundary problem.

We now give the theorems used.

Theorem 3. Let $\Omega_{1}$ and $\Omega_{2}$ in $\mathcal{B}$. Then $\Omega_{1} \cap \Omega_{2} \in \mathcal{B}$.

In order to prove that there is a minimal element in some sense (which we will soon see), we first need a theorem on sequences in $\mathcal{B}$. The two following proofs can be found in [6].

Theorem 4. Let $\left(\Omega_{n}\right)_{n \geq 0}$ be a decreasing sequence of convex domains of $\mathcal{B}$ such that

$$
\Omega:=\bigcap_{n \geq 0}^{\circ} \Omega_{n} \in \mathcal{C} .
$$

Then $\Omega \in \mathcal{B}$.

Proposition 2. Assume that there exists two domains $\Omega_{0} \in \mathcal{A}_{0}$ and $\Omega_{1} \in \mathcal{B}$. We denote by $\mathcal{S}$ the class of domains $D$ such that $\Omega_{0} \subset D$ and $D \in \mathcal{B}$. Then there exists a minimal element in the class $\mathcal{S}$ for the inclusion.

Proof. Even if this result is already proved in [6], we recall it in order to clarify the construction.

Denote by $\mathcal{I}$ the intersection of all domains in the class $\mathcal{S}$, and let

$$
\Omega=\stackrel{\circ}{\mathcal{I}} \text {. }
$$

We claim that $\Omega$ is the minimal elements in $\mathcal{S}$. It suffices to show that $\Omega \in \mathcal{B}$. We observe that we can find a sequence of domains $\left(U_{n}\right)_{n \in \mathbb{N}} \in \mathcal{S}$ such that

$$
\bigcap_{n \geq 0} U_{n}=\mathcal{I}
$$


Consider now the sequence $\left(\Omega_{n}\right)_{n \in \mathbb{N}}$ defined by $\Omega_{1}=D_{1}$ and

$$
\Omega_{n+1}=\Omega_{n} \cap U_{n+1} \text { for all } n \in \mathbb{N} .
$$

By Theorem 3, each $\Omega_{n}$ belongs to $\mathcal{B}$ and by Theorem 4 ,

$$
\Omega:=\overline{\bigcap_{n \geq 0} \Omega_{n}}
$$

belongs to $\mathcal{B}$. Whence the result.

Further on, we will denote by $\Omega$ the minimal element in $\mathcal{S}$. Moreover, a point $x \in \partial \Omega$ is said to be extremal if there is a supporting line $\mathcal{L}$ to $\Omega$ such that $\mathcal{L} \cap \Omega=x$. We denote the set of extremal points on $\partial \Omega \backslash K$ by $E_{\Omega}$. We will now prove that the $u_{\Omega}$ will be in $\mathcal{A}$ as well, but first we need some lemmas, and again, these proofs are the same as in [6].

Lemma 3. Let $x \in \bar{E}_{\Omega} \cap \mathbb{R}_{+}^{n}$. Then

$$
\limsup _{y \rightarrow x}\left|\nabla u_{\Omega}(y)\right|=1 .
$$

This result is already proved in [6]

Lemma 4. Let $\Omega$ be a convex domain, and let $u=u_{\Omega}$. Then for $x \in \partial \mathcal{L}_{t}$,

$$
\limsup _{z \rightarrow x}|\nabla u(z)| \geq \limsup _{z \rightarrow y}|\nabla u(z)|
$$

where $y \in \partial \Omega$ is the point fulfilling

$$
x_{2}(y)=\max _{z \in \partial \Omega} x_{1}(z),
$$

where $x_{1}$ is the direction perpendicular to the tangent plane (or one of them) of $\partial \mathcal{L}_{t}$ at $x_{0}$.

Proof. This is more or less the same arguments as in [6]. We adapt and recall the proof. Suppose that $\partial \mathcal{L}_{t}$ is not $C^{1}$ at $x$. Then by barrier arguments and Lemma 1 , $|\nabla u(x)|=\infty$.

Suppose that $\partial \Omega$ is $C^{1}$ at $y$, and denote by $x_{1}$ the perpendicular direction to $\partial \mathcal{L}_{t}$, such that $x_{1}(x)=0$.

Let

$$
v=u+\left(\limsup _{z \rightarrow y}|\nabla u(z)|-\varepsilon\right) x_{1} .
$$

Then $v$ is harmonic in $\Omega \cap\left\{x_{1}>0\right\}$ and by the maximum principle, $v$ attains it's maximum either at $x$ or at $y$. Suppose that $v$ attains it's maximum at $y$. Then Lemma 1 would imply

$$
\limsup _{z \rightarrow y} \frac{\partial v}{\partial n}(z) \geq 0, \text { and then } \limsup _{z \rightarrow y} \frac{\partial u}{\partial n}(z)+\left(\limsup _{z \rightarrow y}|\nabla u(z)|-\varepsilon\right)\left\langle x_{1}, n\right\rangle \geq 0
$$

where $n$ is the outward pointing normal vector at $y$. As we have seen, $\nabla u$ is perpendicular to the boundary, thus

$$
\limsup _{z \rightarrow y}|\nabla u(z)|=-\limsup _{z \rightarrow y} \frac{\partial u}{\partial n}(z) .
$$

This gives $-\limsup _{z \rightarrow y}|\nabla u(z)|+\left(\limsup _{z \rightarrow y}|\nabla u(z)|-\varepsilon\right)\left\langle x_{1}, n\right\rangle \geq 0$, which together with $\left|\left\langle x_{1}, n\right\rangle\right| \leq 1$ implies $-\varepsilon \geq 0$. Hence, $v$ attains it's maximum at $x$, and by $[7$, Lemma 2.7] we must have

$$
\limsup _{z \rightarrow x} \frac{\partial v}{\partial x_{1}}(z) \leq 0 \text {, i.e. } \limsup _{z \rightarrow x} \frac{\partial u}{\partial x_{1}}(z)+\limsup _{z \rightarrow y}|\nabla u(z)|-\varepsilon \leq 0 .
$$


Moreover,

Thus,

$$
\limsup _{z \rightarrow x}|\nabla u(z)|=-\limsup _{z \rightarrow x} \frac{\partial u}{\partial x_{1}}(z) .
$$

$$
-\limsup _{z \rightarrow x}|\nabla u(z)|+\limsup _{z \rightarrow y}|\nabla u(z)|-\varepsilon \leq 0,
$$

which when $\varepsilon \rightarrow 0$ becomes

$$
\limsup _{z \rightarrow x}|\nabla u(z)| \geq \limsup _{z \rightarrow y}|\nabla u(z)| .
$$

Theorem 5. Assume that there exists two domains $\Omega_{0} \in \mathcal{A}_{0}$ and $\Omega_{1} \in \mathcal{B}$. Then there exists a solution of the free boundary problem $(\mathcal{P})$ in a strong sense.

Proof. The first step of the proof will be to show that $\left|\nabla u_{\Omega}(x)\right| \geq 1$ for all $x \in$ $\partial \Omega \cap \mathbb{R}_{+}^{n}$. By Theorem 2 the level sets $\mathcal{L}_{t}:=\left\{x: u_{\Omega}(x)>t\right\}$, with $0<t<1$, are convex. As a consequence, we can use Lemma 4. The rest of the proof is given by geometric arguments. We denote by $T$ the tangent hyperplane to $\mathcal{L}_{t}$ at $x$. By the property of the level sets, there exists at least one point $y$ in $\overline{E_{\Omega}}$ such that $y$ is at the largest distance to $x$ in a orthogonal direction to $T$. Then, Lemma 1 implies

$$
\left|\nabla u_{\Omega}(x)\right| \geq \limsup _{\substack{z \rightarrow y \\ z \in \Omega}}\left|\nabla u_{\Omega}(z)\right|=1 .
$$

The theorem follows.

\section{Proof of TheOREM 1}

To prove the existence it is by Theorem 5 sufficient to prove that the classes $\mathcal{A}_{0}$ and $\mathcal{B}$ are non-empty.

Proposition 3. The classes $\mathcal{A}_{0}$ and $\mathcal{B}$ are non-empty.

Proof. Let $\Omega \in \mathcal{C}$. By Proposition 1, there is a neighborhood $U$ of $K$ such that $|\nabla u|>\delta$ inside $U \backslash K$. Let $\varepsilon$ be so small that $\{u \geq 1-\varepsilon\} \subset U$, and let

$$
w=\frac{u-(1-\varepsilon)}{\varepsilon} .
$$

Then $w$ is a Dirichlet solution in $\mathcal{L}_{\varepsilon}=\{u \geq 1-\varepsilon\}$ and $|\nabla w|=|\nabla u| / \varepsilon>\delta / \varepsilon$. Moreover, by Lemma 2, $\{u \geq 1-\varepsilon\}$ is convex. Hence, $\{u \geq 1-\varepsilon\} \in \mathcal{A}_{0}$ for $\varepsilon$ small enough.

Now, let $\Omega_{\delta} \in \mathcal{C}$ be a set such that $\partial \Omega_{\delta} \cap\left\{x_{1}=0\right\} \neq K$ and $\operatorname{dist}\left(\partial \Omega_{\delta}, \partial \mathcal{L}_{\varepsilon}\right)<\delta$. Moreover, let $v_{\delta}=u_{\Omega_{\delta}}$. Using that both $w$ and $v_{\delta}$ are harmonic inside $\Omega_{\delta}$ we have, by the maximum principle, that

$$
\left(v_{\delta}-w\right)(x) \leq \delta M\left(r_{0}\right)
$$

for every $x \in \Omega_{\delta}$ outside $N\left(r_{0}\right)$. This implies, by the $C^{1}$-estimate

$$
\left|\nabla v_{\delta}(x)\right| \geq|\nabla w(x)|-\delta M\left(r_{0}\right) C / r>10-\delta M\left(r_{0}\right) C / r,
$$

for some $C>0$, on every ball $B(x, r)$ outside $N\left(r_{0}\right)$, since picking $\delta_{0}$ small enough we will have $\Omega_{\delta} \subset U$ for all $\delta<\delta_{0}$. Picking $\delta$ even smaller, we will at last obtain that $\left|\nabla v_{\delta}\right|>1$ on every compact subset of $\Omega_{\delta}$. Since $\left|\nabla v_{\delta}\right|$ is subharmonic this implies $\Omega_{\delta} \in \mathcal{A}_{0}$ for $\delta$ small enough. 
In order to construct an element in $\mathcal{B}$, let $u_{R}$ be the capacitary potential of the balls $B_{R} / B_{1}$ centered at the origin. That is, except for $n=2, u_{R}$ is given explicitly by

$$
u_{R}=\frac{|x|^{2-n}-R^{2-n}}{R_{0}^{2-n}-R^{2-n}} .
$$

Moreover, denote by $u$ the solution to the Dirichlet problem with $\Omega$ being the upper half disc of $B_{R}$. By the maximum principle, $u_{R} \geq u$ in $\Omega$, and by Lemma 1

$$
\left|\nabla u_{R}\right| \geq|\nabla u|,
$$

on $\partial B_{R} \bigcap\{u>0\}$. Thus, for $R$ big enough we will have $|\nabla u| \leq 1$. Hence, $\mathcal{B}_{0}$ is non-empty.

To prove the regularity we argue by contradiction. Suppose that $\partial \Omega \backslash K$ is not $C^{1}$ at $x$. Then, since $\Omega$ is convex, $\partial \Omega$ has a corner at $x$. But then, by barrier arguments and Lemma 1 we know that

$$
\limsup _{y \rightarrow x}|\nabla(y)|=0,
$$

which is a contradiction. Hence, $\partial \Omega \cap \mathbb{R}_{+}^{n}$ must be $C^{1}$. Hence, we have that the free boundary $(\partial \Omega \backslash K)$ meets the fixed boundary $(K)$ tangentially if the set $(\partial \Omega \backslash K) \cap\left\{x_{1}=0\right\}$ is non-empty. But as we have constructed the element in $\mathcal{A}_{0}$ with $(\partial \Omega \backslash K) \cap\left\{x_{1}=0\right\} \neq \emptyset$ this is clearly true for the minimal element in the class $\mathcal{S}$ as well. Now, applying [4, Thm. 1.4] we obtain that $\partial \Omega$ is $C^{2+\alpha}$ for any $0<\alpha<1$.

The uniqueness follows from the following arguments. Let $u_{1}, \Omega_{1}$ and $u_{2}, \Omega_{2}$ be two different solutions to $(\mathcal{P})$, such that $\Omega_{2} \backslash \Omega_{1} \neq \emptyset$. Then $u_{t}=u_{2}(t x)$ is a solution in

$$
\Omega_{t}=\{x: t x \in \Omega\} .
$$

Moreover, we have $u_{t}=0$ and

$$
\limsup _{y \rightarrow x}\left|\nabla u_{t}(y)\right|=t
$$

on $\partial \Omega_{t} \backslash K_{t}$. Now pick $t>0$ such that there is at least one $x \in \partial \Omega_{t} \backslash K_{t} \cap \partial \Omega_{1} \backslash K$ and such that $\Omega_{t} \subset \Omega$. By the maximum principle we have that $u_{1} \geq u_{t}$ in $\Omega_{t}$. Using Lemma 1 we obtain

$$
1=\limsup _{y \rightarrow x}\left|\nabla u_{1}(y)\right| \geq \limsup _{y \rightarrow x}\left|\nabla u_{t}(y)\right|=t>1,
$$

which is a contradiction. Whence the uniqueness.

\section{Generalizations And COMments}

5.1. Investigation of a limit in the case $n=2$. Obviously, the result is valid if we replace the Bernoulli-type boundary condition with $|\nabla u|=\lambda>0$ as well. Now we will determine the limit of the functions corresponding to $\lambda$ as $\lambda \rightarrow 0$.

Let $\lambda_{n}$ be a decreasing sequence such that $\lambda_{0}=1$ and $\lambda_{n} \rightarrow 0$. Let $u_{n}$ denote the sequence of functions corresponding to the solution with the free boundary condition $\left|\nabla u_{n}\right|=\lambda_{n}$, and let $\Omega_{n}$ be the corresponding domains. Obviously, being a sequence of bounded functions, $u_{n}$ has a subsequential limit. We first observe that as $n \rightarrow \infty, \Omega_{n}$ becomes unbounded in every direction.

Indeed, suppose that $\Omega=\lim \Omega_{n}$ is bounded in some direction. Then we can construct a domain $D$, which is bounded in the same direction such that $\Omega \subset D$ properly. By Lemma 1, $\left|\nabla u_{D}\right|>\delta$ in $D \backslash K$ for some $\delta>0$. Now, let $n$ be big enough such that $\lambda_{n}<\delta$. Then, by taking $D \in \mathcal{A}_{0}\left(\lambda_{n}\right)$ we can construct the minimal element in $\mathcal{B}\left(\lambda_{n}\right)$ as in Proposition 2. By the uniqueness the constructed 
set is nothing else but $\Omega_{n}$. Hence, $\Omega_{n} \subset D$, which is a contradiction. Hence, $\Omega_{n}$ becomes unbounded in every direction as $n \rightarrow \infty$, which implies that $\lim \Omega_{n}=\mathbb{R}_{+}^{2}$.

We now wish to find the limit function, which for sure will fulfill

$$
\begin{cases}u=1 & \text { in } K \\ u=0 & \text { in }\left\{x_{2}=0\right\} \backslash K .\end{cases}
$$

We claim that the function $u(z)=\arg (z+1)-\arg (z-1)$ is the limit of $u_{n}$. Indeed, $u$ fulfills the boundary conditions on $\left\{x_{2}=0\right\}$, and it is harmonic. So, by the properties of harmonic functions in the upper half plane, $u$ is the unique function having the properties above.

5.2. The $p$-Laplacian. In the case of the arbritary $p$-Laplacian there are some small adjustments needed; we will have to replace the fact that $|\nabla u|^{2}$ is subharmonic by [6, Lemma 2.1] and use that the therein defined operator $L_{u}$ admits a maximum principle and that $L_{u}|\nabla u|^{p} \geq 0$. We also have to use that also the $p$-Laplacian admits a maximum and a comparison principle, but in this case this will not make any difference.

Another difference is that the $C^{\infty}$-convergence inside compact subsets has to be replaced by $C^{1, \beta}$-convergence.

In the construction of an element in $\mathcal{A}_{0}$ we use Cauchy's estimate which does not hold for the arbritary $p$-Laplacian. However, this does not cause any difficulties since what we need is just $C^{1}$-convergence inside compact subsets of $\Omega_{\delta}$ which we have in the case of the $p$-Laplacian as well.

Some slight modications are also needed in the construction of an element in $\mathcal{B}$. Here we must use the general expression for the $n$-dimensional radial symmetric $p$-capacitary potential, i.e.

$$
\begin{cases}\frac{|x|^{\frac{p-n}{p-1}}-R^{\frac{p-n}{p-1}}}{R_{p-n}^{p-1}}-R^{\frac{p-n}{p-1}} & \text { for } p \neq n, \\ \frac{\log |x|-\log R}{\log R_{0}-\log R} & \text { for } p=n .\end{cases}
$$

We also remark that the $C^{2+\alpha}$-regularity will not be valid in the general case since the theorem used only applies to elliptic operators. However, the $C^{1}$-regularity will still hold.

5.3. The case of unbounded and irregular $K$. While the irregularity causes some small problems in [6] concerning the lemma corresponding to Lemma 1, irregularities on $\partial K$ would in our case not cause any trouble at all, since we stay away from $\partial K$ in our estimate of $|\nabla u|$.

The case of an unbounded $K$ can be handled in the same way as in [6], and similarly, the uniqueness will not be true in general.

5.4. Usefulness. As mentioned in the introduction, the result in this paper could probably be extended to results similar to those in [8] and [2] for our case. This together with results similar to those in [7] might yield a proof of the existence of solutions to the following problem

$$
\left\{\begin{array}{l}
\Delta_{p} u=f \text { in } \Omega, \\
u=1 \text { on } K \\
u=0 \text { on } \partial \Omega \backslash K,
\end{array}\right.
$$

for certain class of functions $f$. Here $\Omega \subset \mathbb{R}_{+}^{n}$ and $K \subset \partial \Omega$. 


\section{ACKNOWLEDGMents}

We would like to express a great deal of gratitude to Professor Henrik Shahgholian for several light-bringing discussions and for his many useful ideas.

\section{REFERENCES}

1. Andrew Acker, An extremal problem involving distributed resistance, SIAM J. Math. Anal. 12 (1981), 169-172.

2. Andrew Acker, Antoine Henrot, Michael Poghosyan, and Henrik Shahgholian, The multi-layer free boundary problem for the p-Laplacian in convex domains, Interfaces Free Bound. 6 (2004), no. $1,81-103$.

3. Antonio Fasano, «some free boundary problems with industrial applications » in shape optimization a free boundaries, m.c. delfour and g. sabidussi (eds), Graduate Studies in Mathematics, Kluwer Academic Publishers, 1992.

4. Avner Friedman, Variational principles and free-boundary problems, Pure and Applied Mathematics, John Wiley \& Sons Inc., New York, 1982, A Wiley-Interscience Publication.

5. $55-67$.

6. Antoine Henrot and Henrik Shahgholian, Existence of classical solutions to a free boundary problem for the p-Laplace operator. I. The exterior convex case, J. Reine Angew. Math. 521 (2000), 85-97.

7. Existence of classical solutions to a free boundary problem for the p-Laplace operator. II. The interior convex case, Indiana Univ. Math. J. 49 (2000), no. 1, 311-323.

8. - The one phase free boundary problem for the p-Laplacian with non-constant Bernoulli boundary condition, Trans. Amer. Math. Soc. 354 (2002), no. 6, 2399-2416 (electronic).

9. John L. Lewis, Capacitary functions in convex rings, Arch. Rational Mech. Anal. 66 (1977), no. $3,201-224$.

\section{Contact}

\section{Erik Lindgren}

Departement of Mathematics

Royal Institute of Technology

10044 Stockholm

Sweden

Email: eriklin@math.kth.se

Yannick Privat

Institut Élie Cartan de Nancy

Université Henri Poincaré Nancy 1

B.P. 239 , Vandœuvre-lès-Nancy Cedex

France

Email: Yannick.Privateiecn.u-nancy.fr 